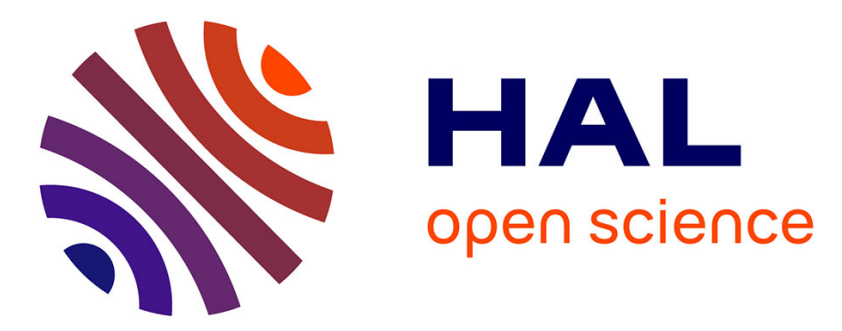

\title{
Capuano Christophe, 2018, Que faire de nos vieux ? Une histoire de la protection sociale de 1880 à nos jours, Paris, Presses de Sciences Po, 352 p.
}

Roméo Fontaine

\section{- To cite this version: \\ Roméo Fontaine. Capuano Christophe, 2018, Que faire de nos vieux ? Une histoire de la protection sociale de 1880 à nos jours, Paris, Presses de Sciences Po, 352 p.. Population (édition française), 2018, pp.597-599. 10.3917/popu.1803.0597 . hal-02079175}

\section{HAL Id: hal-02079175 \\ https://hal.science/hal-02079175}

Submitted on 25 Mar 2019

HAL is a multi-disciplinary open access archive for the deposit and dissemination of scientific research documents, whether they are published or not. The documents may come from teaching and research institutions in France or abroad, or from public or private research centers.
L'archive ouverte pluridisciplinaire HAL, est destinée au dépôt et à la diffusion de documents scientifiques de niveau recherche, publiés ou non, émanant des établissements d'enseignement et de recherche français ou étrangers, des laboratoires publics ou privés. 
CAPUANO Christophe, 2018, Que faire de nos vieux ? Une histoire de la protection sociale de 1880 à nos jours, Paris, Presses de Sciences Po, 352 p.

Au moment où la question de la prise en charge de la perte d'autonomie des personnes âgées revient au premier plan en France, Christophe Capuano retrace l'histoire de la protection sociale des personnes âgées ne pouvant plus réaliser seules les principales activités de la vie quotidienne. À mi-chemin entre les politiques publiques relatives à l'infirmité et celles relatives à la vieillesse, l'ouvrage expose les hésitations des pouvoirs publics, encore aujourd'hui, sur les principes devant guider les politiques de soutien aux personnes âgées en situation de perte d'autonomie.

La première partie de l'ouvrage porte sur la période 1880-1945. L'auteur présente la formation du système d'assistance à l'invalidité ordinaire et la création des prestations sociales répondant spécifiquement aux besoins d'aide à domicile. La seconde période s'étend de 1945 à la fin des années 1960, et voit l'apparition de deux politiques publiques différentes, avec des dispositifs ciblant d'un côté les « grands infirmes » et de l'autre la « vieillesse ». L'auteur s'efforce de montrer que les personnes âgées avec des incapacités peuvent potentiellement bénéficier des deux types de dispositifs, sans pour autant constituer la cible prioritaire de l'une ou de l'autre. La troisième partie enfin s'étend du début des années 1970 jusqu'aujourd'hui. Lauteur insiste sur l'approche sectorielle des politiques sociales qui caractérise cette époque, avec des politiques distinctes dans le champ du handicap, de la vieillesse et de l'emploi, qui apportent des réponses non coordonnées aux besoins des personnes âgées ayant des incapacités. Il discute également de la rupture opérée en 1997 avec la segmentation des populations dépendantes en fonction de l'âge, qui conduit les personnes âgées dépendantes à perdre les bénéfices de la politique contre le handicap. Il tente enfin d'expliquer les échecs des différentes pistes de réformes de la couverture assurantielle du risque dépendance, renvoyant au débat actuel sur le « $5^{\mathrm{e}}$ risque ».

L'évolution des politiques publiques qui tentent de répondre aux besoins d'aide des personnes âgées en perte d'autonomie s'apparente en première lecture à une histoire hésitante, chaotique et peu cohérente sur le temps long. En y introduisant l'évolution des enjeux idéologiques et politiques, ainsi que celle des perceptions des différents acteurs institutionnels et individuels, Christophe Capuano parvient à nous proposer une grille de lecture extrêmement utile de l'évolution de notre système de protection sociale et de ses caractéristiques actuelles.

Quelle que soit l'époque considérée, l'auteur montre la volonté constante des administrations publiques de restreindre les dépenses sociales. Le souci d'économie budgétaire apparaît comme une dimension structurante extrêmement forte des politiques publiques et des principes qui les sous-tendent. Le souci d'économie budgétaire conduira rapidement le décideur public au tournant du $\mathrm{XX}^{\mathrm{e}}$ siècle à inscrire la compensation de l'invalidité ordinaire (hors accident du travail) dans une approche « assistantielle » plutôt qu’ « assurantielle ». 
C'est également cette volonté d'économie qui conduira les décideurs publics, jusqu'à aujourd'hui, à privilégier une politique publique dite de « maintien à domicile » avec la mise en place à partir de 1930 de prestations en espèce pour financer l'aide d'une tierce personne. L'objectif est clair : détourner les personnes en invalidité, les plus de 70 ans en particulier, des hospices ou autres établissements, jugés trop coûteux par les collectivités locales. Tout au long du Xx ${ }^{e}$ siècle, les réformes et revalorisations successives de ces prestations, trop modestes, ne suffiront pas pour donner corps à une politique efficace de maintien à domicile réduisant le nombre d'admissions en institution.

C'est encore la volonté des pouvoirs publics de contenir les dépenses sociales qui conduit à trancher la question de la prise en compte ou non de l'âge dans les politiques d'aide à l'autonomie. Dès le départ, la réponse sera négative, avec des modalités de prise en charge communes aux « infirmes » et aux « vieillards» présentant un même degré d'incapacité. Pendant très longtemps, le choix est donc fait d'assimiler les personnes âgées dites « dépendantes » aux personnes dites « handicapées». L'auteur souligne que ce refus obéissait avant tout à des logiques économiques visant à favoriser le maintien à domicile des populations en perte d'autonomie, solution moins coûteuse que la prise en charge en établissement. C'est à nouveau au nom d'arguments économiques - dispositifs perçus comme trop avantageux pour les personnes âgées avec incapacités et risque de dérapage financier dû au vieillissement de la population- que les personnes âgées avec des incapacités sont distinguées des « personnes handicapées » avec la mise en place de la Prestation spécifique dépendance (PSD) en 1997. Celle-ci marquera donc un tournant car elle opère une distinction juridique des populations avec incapacités en fonction de l'âge. Elle opère également deux ruptures importantes au regard de la tradition française de l'aide à l'autonomie, que l'on peut de nouveau relier à la volonté de contenir les dépenses sociales. Alors que les aides sociales précédentes étaient en espèce, sans contrôle effectif de leur utilisation, la PSD est une prestation affectée à 90 \% à la rémunération de l'aide à domicile. Autre rupture : l'ouverture des droits à la PSD passe dans les mains du financeur (le conseil général du département) alors que l'attribution de l'allocation compensatrice pour tierce personne (ACTP), à laquelle pouvait prétendre les personnes âgées jusque-là, relevait des Commissions techniques d'orientation et de reclassement professionnel (Cotorep), placées sous la tutelle du ministère du Travail.

La question de l'articulation entre les solidarités publiques et les solidarités familiales est le second fil conducteur de l'ouvrage. Christophe Capuano met en avant, sur toute la période considérée, le risque perçu par les pouvoirs publics d'un effet d'éviction des solidarités familiales par les solidarités publiques. Au début du $\mathrm{XX}^{\mathrm{e}}$ siècle, la suspicion vis-à-vis des familles, supposées vouloir se dérober à leurs obligations, donne souvent lieu à de sévères campagnes de contrôle de la mise en œuvre de l'obligation alimentaire légale et à de nombreuses réductions ou radiations de la liste des bénéficiaires de l'assistance sociale. L'augmentation 
du nombre de bénéficiaires de l'assistance ou de l'aide sociale et celle des dépenses sociales seront fréquemment imputées aux familles, soupçonnées de se décharger de leurs proches sur la collectivité. À partir des années 1945, un discours sur le vieillissement démographique se popularise autour d'une lecture catastrophique de ses implications économiques et sociales, alors que se développent en parallèle des discours et des représentations sur le déclin des solidarités familiales envers les ascendants âgés. Outre les évolutions sociodémographiques touchant les structures familiales, ce déclin des solidarités familiales est relié, dans les travaux sociologiques de l'époque, au développement de l'État providence et à la perte de « statut » de la personne âgée induite par la supposée régression des valeurs familiales. Différentes études empiriques mettant en évidence limportance de l'entraide familiale diffuseront petit à petit le paradigme dominant sur l'abandon des vieux par leurs proches. Lauteur y consacre une place importante dans la dernière partie de son ouvrage. La « redécouverte » des solidarités familiales semble finalement apparaître comme une aubaine pour les pouvoirs publics afin de limiter les dépenses publiques. Au tournant des années 2000, le discours sur l'incertitude concernant l'évolution du nombre d'aidants potentiels, leur disponibilité et leur capacité à aider tendra à orienter l'action publique vers un soutien croissant aux aidants.

On ne peut finalement qu'être frappé par la similitude des constats, problématiques et débats actuels avec ceux que retrace l'ouvrage sur un peu plus d'une centaine d'années : l'idéal du vieillissement à domicile, les difficultés financières des services et des établissements médico-sociaux, la pénibilité des conditions de travail, l'instabilité et les difficultés de recrutement, la question des disparités territoriales de prise en charge, celle des indexations des prestations à domicile, la limite d'âge, lévaluation des besoins, la mise en œuvre de l'obligation alimentaire et du recours sur succession, le caractère libératoire ou non des prestations, la question de la rémunération des aidants familiaux, le discours sur la fragilisation de l'aide familiale...

Au moment où la ministre des Solidarités et de la santé a annoncé le lancement d'un débat national associant l'ensemble des acteurs et des citoyens pour aboutir à des propositions début 2019, cet ouvrage offre donc une mise en perspective historique extrêmement précieuse, même si l'histoire de l'aide sociale dont peuvent bénéficier les personnes âgées en établissement et celle du financement public des structures d'hébergement aurait méritée un développement plus conséquent. 